\title{
Medical Management of Intracranial Atheroclerotic Stenosis
}

\author{
Vivien H. Lee*
}

Department of Neurological Sciences, Rush University Medical Center, Chicago, IL 60612, USA

\begin{abstract}
Intracranial atherosclerotic stenosis (ICAS) is a risk factor for ischemic stroke, and the primary aim of management is stroke prevention. The natural history of ICAS is influenced by symptomatic versus asymptomatic status, with symptomatic ICAS having a high recurrent stroke risk. The risk of stroke in symptomatic ICAS is dependent on many factors which will be reviewed in this chapter, including timing after symptoms, degree of stenosis, gender, and possibly other radiographic features (location of stenosis, radiographic progression). Medical management of symptomatic ICAS should include secondary prevention strategies. Based upon the WASID study, aspirin is preferred over Warfarin. Vascular risk factor modification should be promoted in all patients with ICAS to reduce recurrent stroke and vascular events. Despite medical therapy, symptomatic ICAS is associated with a significant recurrent stroke risk.
\end{abstract}

Keywords: Intracranial stenosis, ischemic stroke, WASID.

\section{BACKGROUND}

The major economic burden of intracranial atherosclerotic stenosis is directly related to risk of ischemic stroke, and the primary aim of management is stroke prevention. In the United States, stroke is estimated to occur in more than 700,000 people annually [1]. The anticipated direct and indirect costs of ischemic stroke in the United States have risen to $\$ 58$ billion in 2006 [2, 3]. The Northern Manhattan Stroke Study, a cohort of 438 mixed-ethnicity Manhattan residents, revealed $8 \%$ of acute cerebral infarctions were due to intracranial atherosclerosis [4]. If one conservatively estimates the proportion of ischemic strokes due to intracranial stenosiss to be $8 \%$, the stroke-related economic burden of intracranial atherosclerotic disease is projected to be approximately 5 billion dollars annually.

\section{The Prospective WASID Study}

The Warfarin and Aspirin for Symptomatic Intracranial atheromatous Disease (WASID) study was a prospective, multicenter, randomized trial comparing warfarin and aspirin in patients with symptomatic intracranial stenosiss $(>50 \%$ on cerebral angiogram) [5]. The primary end point was ischemic stroke, brain hemorrhage, or death from vascular causes other than stroke. The aspirin dose was $1300 \mathrm{mg}$ per day and the warfarin dose was adjusted to a target international normalized ratio (INR) between 2 and 3 . WASID was stopped early after enrolling 569 patients due to concerns about safety in the warfarin group. Warfarin was associated with significantly higher rates of adverse events and provided no benefit over aspirin. The primary end point occurred in $22.1 \%$ of the patients in the aspirin group and $21.8 \%$ of those in the warfarin group. Criticisms of the study include the unusual dosing of aspirin $(1300 \mathrm{mg}$ per day) and the fact that patients randomized to warfarin reached target

*Address correspondence to this author at the Department of Neurological Sciences, Rush University Medical Center, Chicago, IL 60612, USA; Tel: 312-942-4500; Fax: 312-563-2206; E-mail: vivien_lee@rush.edu
INR only $63 \%$ of the time [6]. However, the difficulty of maintaining target INR levels is a recognized short-coming inherent to long-term warfarin use that occurs in the clinical practice as well. The conclusion of the WASID study was that aspirin should be used in preference to warfarin for patients with intracranial stenosis. Although it was a negative study, WASID advanced our understanding of symptomatic intracranial stenosis by providing a large subset of medically treated patients with symptomatic intracranial stenosis, from which natural history data and subgroup analysis could be derived.

\section{Warfarin and Basilar Artery Subgroup}

Although the overall WASID study showed no benefit of warfarin over aspirin in symptomatic intracranial stenosis, further analysis was done to evaluate whether certain subgroups benefited from Coumadin. The subset of basilar artery stenosis was the only group that showed decreased rate of primary outcome (ischemic stroke, intracerebral hemorrhage, vascular death) in the warfarin arm compared to the aspirin arm (18\% vs 9\%, p 0.04) [7]. However, many variables were tested without adjusting for multiple comparisons, and the wide confidence intervals and small sample size are also problematic to allow definitive conclusions to be made. The lower rate of primary endpoint in the warfarin arm for the basilar stenosis subgroup is likely a result of chance. Futhermore, there was no significant difference between aspirin and warfarin in the rates of ischemic stroke in the territory of the symptomatic basilar artery. This suggests that warfarin likely has no benefit over aspirin in stroke reduction in patients with basilar artery stenosis.

\section{NATURAL HISTORY}

\section{Asymptomatic Intracranial Stenosis}

Just as asymptomatic extracranial carotid stenosis is wellrecognized to have significantly lower risk of future cerebral ischemia compared to symptomatic carotid disease, asypto- 
matic intracranial stenosis has a lower risk of stroke compared with symptomatic intracranial stenosis. In a prospective study of 102 patients with middle cerebral artery (MCA) stenosis or occlusion, symptomatic MCA disease had an overall stroke risk of $12.5 \%$ per year (ipsilateral 9.1\%), whereas the annual risk for asymptomatic MCA disease was only $2.8 \%$ (ipsilateral $1.4 \%$ ) [8]. There are limited data on the natural history of asymptomatic intracranial stenosis, but most series confirm a relatively low risk of stroke, with an annual ipsilateral stroke risk ranging from 0 to $3.5 \%$ (Table 1). Asymptomatic intracranial stenosis often coexists with symptomatic intracranial stenosis. In theWASID study, coexisting asymptomatic intracranial stenosis was detected in up to $27.3 \%(n=65 / 238)$ of patients with symptomatic intracranial stenosis undergoing MR angiogram (MRA) [9].

\section{Symptomatic Intracranial Stenosis}

The natural history of symptomatic intracranial stenosis can be inferred from clinical trials as well as epidemiology studies categorized by stroke subtype. Data on the stroke subtype of large artery artherosclerosis (LAA) include patients with intracranial stenosis as well as extracranial artery disease. Stroke recurrence, functional outcome, and mortality can be derived from the Rochester, MN stroke epidemiology study [10]. The stroke recurrence rate for the LAA stroke subtype was $18.5 \%$ at 1 -month and $40.2 \%$ at 5 years, which was higher compared to the other subtypes (Table 2) [10]. Good functional outcome (defined as modified Rankin score of 0,1 , or 2) at one-year was observed in $53 \%$ of patients with LAA subtype compared to $82 \%$ of patients with lacunar subtype, and only $27 \%$ in the cardioembolic subtype [10]. The case fatality rate in the LAA cerebral infarction subtype was $8 \%$ at 30 days, and $32 \%$ at 5 years. Interestingly, among the stroke subtypes, LAA had the highest stroke recurrence rate (early and late), but surprisingly had the best long-term survival rates. The LAA subtype of ischemic stroke is associated with better long-term survival than cardioembolic stroke, even when adjusted for age, sex, atrial fibrillation, ischemic heart disease, congestive heart failure, and stroke severity. The limitation of this data is that the category of large-artery atherosclerosis also includes extracranial carotid stenosis.

Studies suggest that symptomatic intracranial stenosis is associated with an annual ipsilateral stroke rate of $7 \%$ to $12 \%$ (Table 1). The Extracranial to Intracranial Bypass Trial evaluated 1377 patients with symptomatic carotid atherosclerotic disease randomized to medical versus bypass surgery followed for an average of 56 months [11]. In the medical group of 714 patients, 59\% were internal carotid artery occlusions. Also comprising the medically treated arm were $24 \%$ with MCA occlusive disease (stenosis and occlusion) and $17 \%$ with intracranial ICA stenosis (above

Table 1. Annual Stroke Rate in Asymptomatic and Symptomatic Intracranial Stenosis

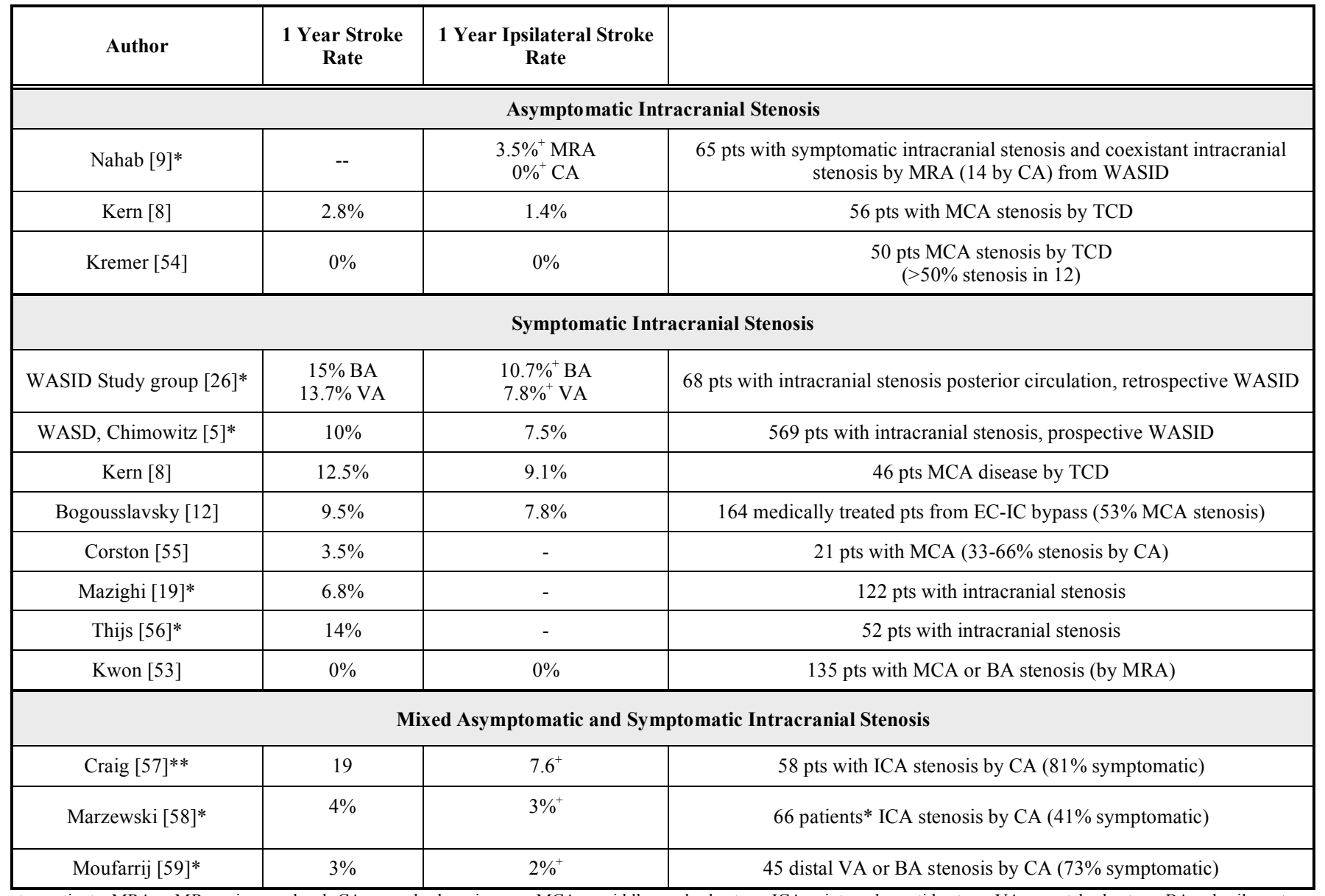

$\mathrm{pt}=$ patients, $\mathrm{MRA}=\mathrm{MR}$ angiogram head, $\mathrm{CA}=$ cerebral angiogram, MCA $=$ middle cerebral artery, ICA $=$ internal carotid artery, $\mathrm{VA}=\mathrm{vertebral}$ artery, $\mathrm{BA}=$ basilar artery, $\mathrm{TCD}=$ Transcranial doppler, ${ }^{+}$in territory of stenosis, $* 50-99 \%$ stenosis, $* *>1 / 3$ stenosis. 
Table 2. Outcome by Cerebral Infarction Subtype [10]

\begin{tabular}{|c|c|c|c|c|}
\hline & Large-Vessel Atherosclerosis & Cardioembolic & Lacunar & Uncertain Cause \\
\hline \multicolumn{5}{|c|}{ Stroke Recurrence } \\
\hline 1 -year & $24.4 \%$ & $13.7 \%$ & $7.1 \%$ & $13.2 \%$ \\
\hline 5 -years & $40.2 \%$ & 31.7 & $24.8 \%$ & $33.2 \%$ \\
\hline 1 -year & $53.4 \%$ & $26.7 \%$ & $81.9 \%$ & $50.3 \%$ \\
\hline \multicolumn{5}{|c|}{ Death Rates } \\
\hline 30-days & $8.1 \%$ & $30.3 \%$ & $1.4 \%$ & $14.0 \%$ \\
\hline
\end{tabular}

the $\mathrm{C}-2$ vertebral body). In the medical arm, $13.8 \%$ suffered stroke or death at the end of one year [11]. In patients with MCA stenosis, the annual stroke rate was $9.5 \%$ and the ipsilateral stroke rate was 7.8\% [12]. This is similar to the natural history data from the WASID trial [13]. In WASID $106(19 \%)$ developed subsequent ischemic stroke, of which $77(14 \%)$ were in the territory of the stenotic artery [14]. The overall rate of ischemic stroke in the territory of the stenotic artery was $11 \%$ at 1 year and $14 \%$ at 2 years [14]. The risk of recurrent stroke is even higher in Asians: in a study conducted by Wong et al. 30\% had recurrent stroke at 2 years of follow up [15].

\section{Degree of Stenosis}

Another significant factor associated with higher subsequent ischemic stroke risk in the territory of the stenotic artery includes degree of stenosis. ${ }^{16}$ Patients with symptomatic intracranial stenosis $\geq 70 \%$ have a higher 2 year risk of stroke compared to patients with $<70 \%$ stenosis $(19 \%$ vs $10 \%)$. If one looks at the high-grade stenosis subgroup ( $>70 \%$ stenosis) with a qualifying event as stroke, the 2 year risk of stroke is in the territory of the artery is $25 \%$ [16]. This data is comparable with symptomatic extracranial carotid artery stenosis in that the degree of stenosis significantly impacts the rate of recurrent ipsilateral stroke with higher risk corresponding to higher degrees of stenosis. Notably, a qualifying event of TIA and stenosis less than $70 \%$ carried about a $3 \%$ and $8 \%$ one and two year respective probability of stroke in the territory of the symptomatic stenotic artery [16]. The risk of ipsilateral stroke in extracranial carotid stenosis has a U-shape curve, with risk peaking at a high degree of stenosis with subsequent decrease in near-occlusion and occlusion [17].

\section{Timing}

The WASID study also demonstrated that recent symptoms were associated with a higher risk for subsequent stroke in patients with symptomatic intracranial stenosis [16]. The majority (78\%) of recurrent strokes occurred within the first year [16]. In the $>70 \%$ stenosis subgroup,
$23 \%$ of the stroke risk was in the $1^{\text {st }}$ year, with only an additional $2 \%$ risk in the second year. Patients with recent symptoms ( $\leq 17$ days) had a higher risk of 2-year stroke compared to patients without recent symptoms $(17 \%$ vs $10 \%$ ) [16]. In the 36 patients with symptomatic vertebrobasilar (VB) stenosis (50-99\%), the recurrent stroke risk was $13.8 \%(5 / 36)$ at 90 days, and most recurrences occurred within 30 days after the initial event [18]. The GESICA study found that recurrent cerebral ischemic events occurred at a median time of 2 months following the qualifying event [19]. Therefore, patients are likely at highest risk soon after their qualifying event, and the recurrent stroke risk drops dramatically thereafter for patients who are 1 year out from their event.

\section{Qualifying Event}

Type of qualifying event (TIA versus stroke) was not associated with recurrent risk of stroke in the territory of the stenosis. However, NIH Stroke scale $>1$, which strongly correlated with type of qualifying event, was associated with an increase in risk of recurrent stroke in the territory of the symptomatic vessels [16]. The reasons for this apparent difference are unclear.

\section{Sex}

Sex differences in stroke risk are demonstrable in population-based studies, with men having 4 times higher incidence rate of first ischemic stroke due to large-vessel atherosclerosis than women [20]. However, in intracranial stenosis, women may have greater recurrent stroke risk than men. A subgroup analaysis of WASID showed that women with symptomatic intracranial stenosis were at significantly higher risk for recurrent ischemic stroke and for the combined end point of stroke or vascular death comared with men (2-year rates $28.4 \%$ versus $16.6 \%, p=0.017$ ) [21]. This increased risk persists even after adjusting for sociodemographic features, lifestyle, vascular risk factors, angiographic findings, and features of the qualifying event [21]. Female sex was also a signficant risk factor associated with subsequent ischemic stroke in the territory of the 
stenotic intracranial artery [16]. The reason for sex disparity is unclear and is likely multifactorial.

\section{Radiographic Progression of Stenosis}

Radiographic progression of intracranial stenosis is common. Among 21 patients with 45 intracranial stenoses ( $48 \%$ had presented with stroke), repeat angiography done at a mean of 26.7 months demonstrated an increase in the mean stenosis of intracranial lesions from $43.9 \%$ to $51.8 \%$ ( $\mathrm{p}=$ 0.03 ) [22]. There were 4 TIAs and no strokes in the followup period of this study. Based upon a $10 \%$ change, $40 \%$ of the intracranial stenoses progressed, $40 \%$ were stable, and $20 \%$ regressed [22]. Progression of symptomatic intracranial stenosis has been suggested to predict patients who are at increased risk of recurrent clinical events. Among 40 patients with angiogram confirmed symptomatic MCA stenosis followed by TCD for a median of 26 months, onethird progressed radiographically and $60 \%$ remained stable. Progression of MCA stenosis as detected by TCD was independently associated with recurrent ischemic events in the territory of the MCA stenosis and occurred in 8 patients (20\%). Although intriguing, the significance of these findings should be interpreted with caution given the relatively small numbers [23].

\section{Location of Stenosis}

Although the overall stroke recurrence is similar following anterior and posterior circulation symptomatic intracranial stenosis, some studies have suggested that the early stroke risk may be higher in patients with VB stenosis. The "higher" early stroke risk may be due to a higher prevalence of large artery stenosis in patients with posterior circulation events compared with carotid territory events [24, 25]. In a population based study, 37/141(26.2\%) symptomatic patients with VB events were found to have > $50 \%$ vertebral or basilar stenosis compared with only $41 / 357(11.5 \%)$ of anterior circulation event patients found to have carotid stenosis [25]. Furthermore, 17/37 (46\%) patients who had symptomatic VB stenosis had a recurrent TIA or ischemic stroke in the posterior circulation within 90 days compared with $22 / 104$ (21\%) patients without VB stenosis [25]. A subgroup analysis of patients with symptomatic intracranial VB stenosis in the retrospective WASID study associated VB stenosis with a high risk of recurrent stroke (22\%) [26]. However, in the prospective WASID study, the location of stenosis (anterior versus posterior) was not a significant factor in the risk of recurrent stroke in the territory of the stenotic artery [16]. Lastly, the presence of multiple or tandem intracranial, extracranial carotid, or vertebral artery atherosclerotic lesions may also be associated with poor outcome [27].

Other factors not significantly associated with an increased risk of subsequent ischemic stroke in the territory of the stenotic artery include length of stenosis, use of antithrombotic medication at the time of the qualifying event, and medication treatment (aspirin versus warfarin) [5, 16]. In the WASID study, blacks more frequently achieved an endpoint of stroke, intracerebral hemorrhage, or vascular death compared to whites ( 28 vs 20\%): the recurrence rate for ischemic stroke in blacks was $25 \%$ compared to $16 \%$ for whites [28].

\section{MEDICAL MANAGEMENT}

\section{Acute Therapy}

Medical treatment should be directed by the general principles stated by the American Heart Association (AHA) that include permissive hypertension in the immediate poststroke period and treating and correcting elevated blood sugar, fever, hypoxia, and hypotension [29]. Intravenous tissue plasminogen activator (IV tPA) should be considered for all subtypes of acute ischemic stroke. Unadjusted outcomes demonstrated a trend for better outcomes following lacunar strokes than cardioembolic or large artery strokes, but this difference in treatment response disappeared after adjusting for baseline National Institute Health Stroke Score. Thus, IV tPA treatment is considered standard of care for all stroke subtypes, including intracranial stenosis [3032]. Chinese Acute Stroke Trial and The International Stroke Trial were 2 large trials that showed a modest but significant benefit when treatment with aspirin was initiated within 48 hours of stroke [33, 34]. Aspirin remains the only oral antiplatelet agent that has been evaluated for the treatment of acute ischemic stroke. Data on clopidogrel or combination aspirin and dipyridamole for treatment of acute ischemic stroke are not available.

\section{Secondary Stroke Prevention}

Specific risk factors have been associated with an increased risk of vascular events among patients intracranial stenosis [35]. In the WASID study, elevated systolic blood pressure (SBP) $(\geq 140 \mathrm{~mm} \mathrm{Hg})$ and cholesterol levels $(\geq 200$ $\mathrm{mg} / \mathrm{dL}$ ) were associated with an increased risk of major vascular event (stroke, myocardial infarction, vascular death) in symptomatic intracranial stenosis [35]. The clinical practice of maintaining high blood pressure in patients with intracranial stenosiss to maintain perfusion is not recommended given the WASID subgroup analysis which found higher blood pressure is associated with increased risk of recurrent ischemic stroke [36]. This increased stroke risk in the territory of the stenotic artery persists even after adjustment for other risk factors and was particularly notable in those with SBP $>160 \mathrm{mmHg}$ [36]. However, the optimal timing of the blood pressure lowering, remains uncertain and a subset of patients may benefit from permissive hypertension in the acute and early subacute periods following the event.

There is scant information available on the impact of other risk factors (such as diabetes or tobacco use) on risk of recurrent events in symptomatic intracranial stenosis. In WASID, a hemoglobin A1c $>7 \%$ was associated with higher risk of major vascular event (31\% vs 20\%). This difference was not significant, likely due to low statistical power [35]. However, risk factor modification strategies and goals can be extrapolated from those found to be beneficial in symptomatic extracranial carotid stenosis. Hypertension, smoking, and diabetes are established risk factors for atherosclerosis and have been demonstrated in many studies $[37,38]$. 
Table 3. AHA/ASA Recommendations for Secondary Stroke Prevention $[50,51]$

\begin{tabular}{|c|c|c|}
\hline Risk Factor & Recommendation & Level of Evidence \\
\hline \multicolumn{3}{|c|}{ Modifiable Behavior Risk Factors } \\
\hline Smoking & $\begin{array}{l}\text { All ischemic stroke or TIA patients who have smoked in the past year should be strongly encouraged not to smoke. } \\
\qquad \text { Avoid environmental smoke. } \\
\text { Counseling, nicotine products, and oral smoking cessation medications have been found to be effective for smokers. }\end{array}$ & $\begin{array}{l}\text { Class I, Level C } \\
\text { Class IIa, Level C } \\
\text { Class IIa, Level B }\end{array}$ \\
\hline Alcohol & $\begin{array}{l}\text { Patients with prior ischemic stroke or TIA who are heavy drinkers should eliminate or reduce their consumption of } \\
\text { alcohol. } \\
\text { Light to moderate levels of } \leq 2 \text { drinks per day for men and } 1 \text { drink per day for nonpregnant women may be considered. }\end{array}$ & $\begin{array}{l}\text { Class I, Level A } \\
\text { Class IIb, Level C }\end{array}$ \\
\hline Obesity & $\begin{array}{l}\text { Weight reduction may be considered for all overweight ischemic stroke or TIA patients to maintain the goal of a BMI of } \\
18.5 \text { to } 24.9 \mathrm{~kg} / \mathrm{m} 2 \text { and a waist circumference of }<35 \text { in for women and }<40 \text { in for men. Clinicians should encourage } \\
\text { weight management through an appropriate balance of caloric intake, physical activity, and behavioral counseling. }\end{array}$ & Class IIb, Level C \\
\hline $\begin{array}{l}\text { Physical } \\
\text { activity }\end{array}$ & $\begin{array}{c}\text { For those with ischemic stroke or TIA who are capable of engaging in physical activity, at least } 30 \text { minutes of moderate- } \\
\text { intensity physical exercise most days may be considered to reduce risk factors and comorbid conditions that increase the } \\
\text { likelihood of recurrence of stroke. For those with disability after ischemic stroke, a supervised therapeutic exercise } \\
\text { regimen is recommended. }\end{array}$ & Class IIb, Level \\
\hline
\end{tabular}

Treatable Vascular Risk Factors \begin{tabular}{|c|c|c|c|c|}
\hline Hypertension & $\begin{array}{r}\text { Antihypertensive treatment is recommended for prevention of recurrent stroke and other vascular events in persons who } \\
\text { have had an ischemic stroke and are beyond the hyperacute period. }\end{array}$
\end{tabular}

Because this benefit extends to persons with and without a history of hypertension, this recommendation should be considered for all ischemic stroke and TIA patients.

An absolute target BP Level And reduction are uncertain and should be individualized, but benefit has been associated with an average reduction of $\sim 10 / 5 \mathrm{~mm} \mathrm{Hg}$ and normal BP levels have been defined as $<120 / 80$ by JNC-7.

Several lifestyle modifications have been associated with BP reductions and should be included as part of a comprehensive approach antihypertensive therapy.

Optimal drug regimen remains uncertain; however, available data support the use of diuretics and the combination of diuretics and an ACEI. Choice of specific drugs and targets should be individualized on the basis of reviewed data and consideration, as well as specific patient characteristics (eg, extracranial cerebrovascular occlusive disease, renal impairment, cardiac disease, and DM).

Diabetes $\quad$ More rigorous control of blood pressure and lipids should be considered in patients with diabetes.

Although all major classes of antihypertensives are suitable for the control of BP, most patients will require $>1$ agent. ACEIs and ARBs are more effective in reducing the progression of renal disease and are recommended as first-choice medications for patients with DM.

Glucose control is recommended to near-normoglycemic levels among diabetics with ischemic stroke or TIA to reduce microvascular complications.

The goal for $\mathrm{Hb}$ Alc should be $\leq 7 \%$.

Cholesterol

Ischemic stroke or TIA patients with elevated cholesterol, comorbid coronary artery disease, or evidence of an atherosclerotic origin should be managed according to NCEP III guidelines, which include lifestyle modification, dietary guidelines, and medication recommendations.

Statin agents are recommended, and the target goal for cholesterol lowering for those with CHD or symptomatic atherosclerotic disease is an LDL-C level of $<100 \mathrm{mg} / \mathrm{dL}$. An LDL-C $<70 \mathrm{mg} / \mathrm{dL}$ is recommended for very high-risk persons with multiple risk factors.

On the basis of the SPARCL trial, administration of statin therapy with intensive lipid- lowering effects is recommended for patients with atherosclerotic ischemic stroke or TIA and without known CHD to reduce the risk of stroke and cardiovascular events.

Ischemic stroke or TIA patients with low HDL cholesterol may be considered for treatment with niacin or gemfibrozil.

\section{Recommendations for Anti-Platelet Therapy}

For patients with noncardioembolic ischemic stroke or TIA, antiplatelet agents rather than oral anticoagulation are recommended to reduce the risk of recurrent stroke and other cardiovascular events.

Aspirin (50 to $325 \mathrm{mg} / \mathrm{d}$ ) monotherapy, the combination of aspirin and extended-release dipyridamole, and clopidogrel monotherapy are all acceptable options for initial therapy.

The combination of aspirin and extended-release dipyridamole is recommended over aspirin alone.

Clopidogrel may be considered over aspirin alone on the basis of direct-comparison trials.

For patients allergic to aspirin, clopidogrel is reasonable.

The addition of aspirin to clopidogrel increases the risk of hemorrhage. Combination therapy of aspirin and clopidogrel is not routinely recommended for ischemic stroke or TIA patients unless they have a specific indication for this therapy.
Class I, Level A

Class IIa, Level B

Class IIa, Level B

Class IIb, Level C

Class I, Level A

Class IIa, Level B

Class I, Level A

Class I, Level A

Class IIa, Level B

Class I, Level A

Class I, Level A

Class I, Level B

Class IIb, Level B

Class I, Level A

Class I, Level A

Class I, Level B

Class IIb, Level B

Class IIa, Level B 
Studies of patients with extracranial carotid disease have consistently shown that independent risk factors for carotid atherosclerosis include age, cigarette smoking, hypertension, diabetes mellitus and elevated cholesterol [39-42]. The presence of risk factors and severity of these risk factors negatively impact the progression of carotid disease. Progression of carotid atherosclerotic disease by ultrasound is associated with smoking, hypertension, and diabetes [43]. The severity of diabetes and hypertension (and therefore control of disease) can influence progression of carotid atherosclerosis as measured by carotid intima-media thickness (IMT) on ultrasound. Type 1 diabetes assigned to intensive insulin therapy in the Diabetes Control and Complications Trial had decreased progression of carotid IMT after 6 years compared with those assigned to conventional diabetes treatment [44]. A population-based epidemiology study evaluated 1198 residents in Beijing and revealed that the degree of hypertension as well as duration of hypertension are associated with subclinical carotid atherosclerosis [45]. A recent meta-analysis reported that each $10 \%$ reduction in low-density lipoprotein cholesterol reduced the risk of stroke by $15.6 \%$ and carotid IMT by $0.73 \%$ per year, this supports the suggestion that statin use may reduce progression of carotid atherosclerosis $[46,47]$.

Vascular risk factor modification should be considered in all patients with intracranial stenosis to reduce progression of disease and overall cardiovascular risk. Indeed, intracranial stenosis is also a marker for systemic atherosclerosis such as coronary artery disease and aortic plaques [48, 49]. In fact, the risk of coronary artery disease, myocardial infarction, and vascular death among patients with intracranial stenosis was confirmed and verified in numerous studies [5, 11, 49].

American Heart Association secondary stroke prevention guidelines should be followed in all stroke subtypes, including intracranil stenosis (Table 3). For secondary stroke prevention, aspirin, aspirin plus extended release dipyridamole, or clopidogrel monothreapy are all acceptable options for initial antiplatelet medications for secondary stroke prevention $[50,51]$. Warfarin is not a safe option given the results of WASID [5]. Alternate antiplatelet medication choices are reasonable but have not been specifically studied in intracranial stenosis patients. Dual anti-platelet medication use (aspirin plus clopidogrel) has been shown to have no benefit in secondary stroke prevention and therefore is generally not recommended for intracranial stenosis [52]. Data on novel antiplatelet agents such as cilostazol, a phosphodiesterase inhibitor, remains scant and use in the routine clinical setting is not currently supported by evidence-based medicine [53].

Despite medical therapy, symptomatic intracranial stenosis is associated with a high recurrent stroke risk. Interventional treatments are currently being studied as additional treatment options to improve outcomes.

\section{CONCLUSIONS}

The prevalence of intracranial stenosis among stroke patients is approximately $8 \%$. The annual ipsilateral stroke risk for people with asymptomatic intracranial stenosis is approximately $1.4-3.5 \%$, and for patients with symptomatic intracranial atherosclerosis is approximately $12 \%$. This stroke risk is substantially increased in specific subgroup of patients such as stenosis $\geq 70 \%$, recent symptomatology, and women. For example, in patients with stenosis $\geq 70 \%$ with symptoms within 30 days of the qualifying event, the 1 year ipsilateral stroke risk may be as high as $29 \%$. The stroke risk in symptomatic intracranial stenosis is not uniformly distributed over time, but is much higher during the acute period after an event and approaches $20 \%$ at 1-month after initial cerebral infarction. Other factors such as radiographic progression of stenosis, type of qualifying event, and location of stenosis, have been considered possible risk factors of recurrent stroke; however, supporting evidence from prospective studies is lacking.

Management of acute stroke due to intracranial stenosis should include consideration of IV tPA for eligible patients and aspirin for those not eligible for thrombolysis. In secondary prevention, warfain is associated with an increased bleeding risk and provides no benefit over aspirin: thus in patients with symptomatic intracranial atherosclerosis aspirin use in recommended. Alternate antiplatelet medication choices are reasonable but have not been specifically studied in this stroke subtype. Hypertension and hyperlipidemia are specifically associated with increased recurrent stroke risk in symptomatic intracranial stenosis. Vascular risk factor modification should be promoted in all patients with intracranial stenosis to reduce recurrent stroke and vascular events. Despite medical therapy, symptomatic intracranial stenosis is associated with a high recurrent stroke risk. Interventional treatments are currently being studied as additional treatment options to decrease the risk of recurrent stroke

\section{REFERENCES}

[1] Broderick J, Brott $\mathrm{T}$, Kothari $\mathrm{R}$, et al. The Greater Cincinnati/Northern Kentucky Stroke Study: preliminary first-ever and total incidence rates of stroke among blacks. Stroke 1998; 29: 415-21.

[2] Taylor TN, Davis PH, Torner JC, Holmes J, Meyer JW, Jacobson MF. Lifetime cost of stroke in the United States. Stroke 1996; 27: 1459-66.

[3] Thom $\mathrm{T}$, Haase N, Rosamond $\mathrm{W}$, et al. American Heart Association Statistics Committee and Stroke Statistics Subcommittee. Heart disease and stroke statistics--2006 update: a report from the American Heart Association Statistics Committee and Stroke Statistics Subcommittee. Circulation 2006; 113: e85151 .

[4] Sacco RL, Kargman DE, Gu Q, Zamanillo MC. Race-ethnicity and determinants of intracranial atherosclerotic cerebral infarction. The Northern Manhattan Stroke Study. Stroke 1995; 26: 14-20.

[5] Chimowitz MI, Lynn MJ, Howlett-Smith H, et al. Warfarin-Aspirin Symptomatic Intracranial Disease Trial Investigators. Comparison of warfarin and aspirin for symptomatic intracranial arterial stenosis. N Engl J Med 2005; 352: 1305-16.

[6] Koroshetz WJ. Warfarin, aspirin, and intracranial vascular disease. N Engl J Med 2005; 352: 1368-70.

[7] Kasner SE, Lynn MJ, Chimowitz MI, et al. For the for the Warfarin Aspirin Symptomatic Intracranial Disease (WASID) Trial Investigators. Warfarin $v s$ aspirin for symptomatic intracranial stenosiss: Subgroup analyses from WASID. Neurology 2006; 67; 1275-8.

[8] Kern R, Steinke W, Daffertshofer M, Prager R, Hennerici M. Stroke recurrences in patients with symptomatic $v s$ asymptomatic middle cerebral artery disease. Neurology 2005; 65: 859-864.

[9] Nahab F, Cotsonis G, Lynn M, et al. For the WASID study group. Prevalence and prognosis of coexistent asymptomatic intracranial stenosiss. Stroke 2008; 39; 1039-41.

[10] Petty GW, Brown RD Jr, Whisnant JP, Sicks JD, O'Fallon WM, Wiebers DO. Ischemic stroke subtypes: a population-based study 
of functional outcome, survival, and recurrence. Stroke 2003; 31 : 1062-8.

[11] The EC/IC Bypass Study Group. Failure of extracranial-intracranial arterial bypass to reduce the risk of ischemic stroke: results of an international randomized trial. N Engl J Med 1985; 313: 11912000 .

[12] Bogousslavsky J, Barnett HJ, Fox AJ, Hachinski VC, Taylor W. Atherosclerotic disease of the middle cerebral artery. Stroke 1986; 17: 1112-20.

[13] WASID Trial Investigators. Comparison of warfarin and aspirin for symptomatic intracranial arterial stenosis. N Engl J Med 2005; 352 : 1305-16.

[14] Famakin BM, Chimowitz MI, Lynn MJ, Stern BJ, George MG. For the WASID Trial Investigators. Causes and severity of ischemic stroke in patients with symptomatic intracranial arterial stenosis. Stroke 2009; 40: 1999-2003.

[15] Wong LKS. Global burden of intracranial atherosclerosis. Int J Stroke 2006; 1: 158-9.

[16] Kasner SE, Chimowitz MI, Lynn MJ, et al. Warfarin Aspirin Symptomatic Intracranial Disease Trial Investigators. Predictors of ischemic stroke in the territory of a symptomatic intracranial arterial stenosis. Circulation 2006; 113: 555-63.

[17] Inzitari D, Eliasziw M, Gates P, et al. The causes and risk of stroke in patients with asymptomatic internal-carotid-artery stenosis. North American Symptomatic Carotid Endarterectomy Trial Collaborators. N Engl J Med 2000; 342: 1693-700.

[18] Gulli G, Khan S, Markus HS. Vertebrobasilar stenosis predicts high early recurrent stroke risk in posterior circulation stroke and TIA. Stroke 2009; 40: 2732-7.

[19] Mazighi M, Tanasescu R, Ducrocq X, et al. Prospective study of symptomatic atherothrombotic intracranial stenoses: The GESICA Study. Neurology 2006; 66: 1187-91.

[20] Petty GW, Brown RD Jr, Whisnant JP, Sicks JD, O'Fallon WM, Wiebers DO. Ischemic stroke subtypes: a population-based study of incidence and risk factors. Stroke 1999; 30: 2513-6.

[21] Williams JE, Chimowitz MI, Cotsonis GA, Lynn MJ, Waddy SP. For the WASID investigators. Gender differences in outcomes among patients with symptomatic intracranial arterial stenosis. Stroke 2007; 38; 2055-62.

[22] Akins PT, Pilgram TK, Cross DT, Moran CJ. Natural history of stenosis from intracranial atherosclerosis by serial angiography. Stroke 1998; 29; 433-8.

[23] Arenillas JF, Molina CA, Montaner J, Abilleira S, GonzálezSánchez MA, Álvarez-Sabín J. Progression and clinical recurrence of symptomatic middle cerebral artery stenosis: a long-term followup transcranial Doppler ultrasound study. Stroke 2001; 32; 2898904.

[24] Flossmann E, Rothwell PM. Prognosis of vertebrobasilar transient ischaemic attack and minor stroke. Brain 2003; 126: 1940-54.

[25] Marquardt L, Kuker W, Chandratheva A, Geraghty O, Rothwell PM. Incidence and prognosis of $>50 \%$ symptomatic vertebral or basilar artery stenosis: prospective population-based study. Brain 2009: 132; 982-8

[26] The WASID study group. Prognosis of patients with symptomatic vertebral or basilar artery stenosis. The warfarin-aspirin symptomatic intracranial disease (WASID) study group. Stroke 1998; 29: 1389 -92.

[27] Glass TA, Hennessey PM, Pazdera L, et al. Outcome at 30 days in the New England Medical Center Posterior Circulation Registry. Arch Neurol 2002; 59: 369-76.

[28] Waddy SP, Cotsonis G, Lynn MI, et al. Racial differences in vascular risk factors and outcomes of patients with intracranial atherosclerotic arterial stenosis. Stroke 2009; 40: 719-25.

[29] Adams HP, del Zoppo G Jr, Alberts MJ, et al. Guidelines for the early management of adults with ischemic stroke: a guideline from the American Heart Association/American Stroke Association Stroke Council, Clinical Cardiology Council, Cardiovascular Radiology and Intervention Council, and the atherosclerotic peripheral vascular disease and quality of care outcomes in research interdisciplinary working groups: The American Academy of Neurology affirms the value of this guideline as an educational tool for neurologists. Stroke 2007; 38; 1655-711.

[30] Hsia AW, Sachdev HS, Tomlinson J, Hamilton SA, Tong DC. Efficacy of IV tissue plasminogen activator in acute stroke: Does stroke subtype really matter? Neurology $2003 ; 61 ; 71-75$.
[31] National Institute of Neurological Disorders and Stroke rtPA Stroke Study Group. Tissue plasminogen activator for acute ischemic stroke. N Engl J Med 1995; 333: 1581-7.

[32] National Institute of Neurological Disorders and Stroke rtPA Stroke Study Group. Generalized efficacy of rt-PA for acute stroke. Subgroup analysis of the NINDS rt-PA stroke trial. Stroke 1997; 28: 2119-25.

[33] CAST (Chinese Acute Stroke Trial) Collaborative Group. CAST: randomized placebo-controlled trial of early aspirin use in 20,000 patients with acute ischaemic stroke. Lancet 1997; 349: 1641-9.

[34] International Stroke Trial Collaborative Group. The International Stroke Trial (IST): a randomised trial of aspirin, subcutaneous heparin, both, or neither among 19435 patients with acute ischaemic stroke. Lancet 1997; 349: 1569-81.

[35] Chaturvedi S, Turan TN, Lynn MJ, et al. Risk factor status and vascular events in patients with symptomatic intracranial stenosiss. Neurology 2007; 69: 2063-8.

[36] Turan TN, Cotsonis G, Lynn MJ, Chaturvedi S, Chimowitz M. For the Warfarin-Aspirin Symptomatic Intracranial Disease (WASID) trial investigators. Relationship between blood pressure and stroke recurrence in patients with intracranial arterial stenosis. Circulation 2007; 115; 2969-75.

[37] O'Leary DH, Polak JF, Kronmal RA, et al. Distribution and correlates of sonographically detected carotid artery disease in the Cardiovascular Health Study. The CHS Collaborative Research Group. Stroke 1992; 23: 1752-60.

[38] Wilson PW, Hoeg JM, D'Agostino RB, et al. Cumulative effects of high cholesterol levels, high blood pressure, and cigarette smoking on carotid stenosis. N Engl J Med 1997; 337: 516-22.

[39] Fine-Edelstein JS, Wolf PA, O'Leary DH, et al. Precursors of extracranial carotid atherosclerosis in the Framingham Study. Neurology 1994; 44: 1046-50.

[40] Whisnant JP, Homer D, Ingall TJ, Baker HL Jr, O'Fallon WM, Wiebers DO. Duration of cigarette smoking is the strongest predictor of severe extracranial carotid artery atherosclerosis. Stroke 1990; 21: 707-14.

[41] Ingall TJ, Homer D, Baker HL Jr, Kottke BA, O'Fallon WM, Whisnant JP. Predictors of intracranial carotid artery atherosclerosis. Duration of cigarette smoking and hypertension are more powerful than serum lipid levels. Arch Neurol 1991; 48: 687-91.

[42] O'Leary DH, Anderson KM, Wolf PA, Evans JC, Poehlman HW. Cholesterol and carotid atherosclerosis in older persons: the Framingham Study. Ann Epidemiol 1992; 2: 147-53.

[43] Roederer GO, Langlois YE, Jager KA, et al. The natural history of carotid arterial disease in asymptomatic patients with cervical bruits. Stroke 1984; 15: 605-13.

[44] Nathan DM, Lachin J, Cleary P, et al. Diabetes control and complications trial. Epidemiology of diabetes interventions and complications research group. Intensive diabetes therapy and carotid intima-media thickness in type 1 diabetes mellitus. $\mathrm{N}$ Engl $\mathrm{J}$ Med 2003; 348: 2294-303.

[45] Lu M, Wu L, Shi P, Kang S, Shi L, Wu Y. Hypertension and subclinical carotid atherosclerosis in a suburban general population in China. J Hyperten 2004; 22: 1699-706.

[46] Amarenco P, Labreuche J, Lavallee P, Touboul PJ. Statins in stroke prevention and carotid atherosclerosis: systematic review and upto-date meta-analysis. Stroke 2004; 35: 2902-9.

[47] Kang S, Wu Y, Li X. Effects of statin therapy on the progression of carotid atherosclerosis: a systematic review and meta-analysis. Atherosclerosis 2004; 177: 433-42.

[48] Uehara T, Tabuchi M, Hayashi T, Kurogane H, Yamadori A. Asymptomatic occlusive lesions of carotid and intracranial arteries in Japanese patients with ischemic heart disease. Stroke 1996; 27 293-7.

[49] Wong KS, Ng PW, Tang A, Liu R, Yeung V, Tomlinson B. Prevalence of asymptomatic intracranial atherosclerosis in highrisk patients. Neurology 2007; 68: 2035-8.

[50] Sacco RL, Adams R, Albers G, et al. American Heart Association; American Stroke Association Council on Stroke; Council on Cardiovascular Radiology and Intervention; American Academy of Neurology. Guidelines for prevention of stroke in patients with ischemic stroke or transient ischemic attack: a statement for healthcare professionals from the American Heart Association/ American Stroke Association Council on Stroke: co-sponsored by the Council on Cardiovascular Radiology and Intervention: the 
American Academy of Neurology affirms the value of this guideline. Stroke 2006; 37: 577-617.

[51] Adams RJ, Albers G, Alberts MJ, et al. Update to the AHA/ASA Recommendations for the prevention of stroke in patients with stroke and transient ischemic attack. Stroke 2008; 39; 1647-52.

[52] Diener HC, Bogousslavsky J, Brass LM, et al. On behalf of the MATCH investigators. Aspirin and clopidogrel compared with clopidogrel alone after recent ischaemic stroke or transient ischaemic attack in high-risk patients (MATCH): randomised, double-blind, placebo-controlled trial. Lancet 2004; 364: 331-7.

[53] Kwon SU, Cho YJ, Koo JS, et al. Cilostazol prevents the progression of the symptomatic intracranial arterial stenosis: the multicenter double-blind placebo-controlled trial of cilostazol in symptomatic intracranial arterial stenosis. Stroke 2005; 36; 782-6.

[54] Kremer C, Schaettin T, Georgiadis D, Baumgartner RW. Prognosis of asymptomatic stenosis of the middle cerebral artery. J Neurol Neurosurg Psychiatry 2004; 75: 1300-3.
[55] Corston RN, Kendall BE, Marshall J. Prognosis in middle cerebral artery stenosis. Stroke 1984; 15: 237-41.

[56] Thijs VN, Albers GW. Symptomatic intracranial atherosclerosis Outcome of patients who fail antithrombotic therapy. Neurology 2000; 55: 490-7.

[57] Craig DR, Meguro K, Watridge C, Robertson JT, Barnett HJ, Fox AJ. Intracranial internal carotid artery stenosis. Stroke 1982; 13: 825-8.

[58] Marzewski DJ, Furlan AJ, St Louis P, Little JR, Modic MT, Williams G. Intracranial internal carotid artery stenosis: longterm prognosis. Stroke 1982; 13: 821-4

[59] Moufarrij NA, Little JR, Furlan AJ, Leatherman JR, Williams GW. Basilar and distal vertebral artery stenosis: long-term follow-up. Stroke 1986; 17: 938-42.

(C) Vivien H. Lee; Licensee Bentham Open.

This is an open access article licensed under the terms of the Creative Commons Attribution Non-Commercial License (http://creativecommons.org/licenses/by$\mathrm{nc} / 3.0 /$ ) which permits unrestricted, non-commercial use, distribution and reproduction in any medium, provided the work is properly cited. 